\title{
Helicobacter pylori infection as a protective factor against multiple sclerosis risk in females
}

\author{
Marzena J Fabis Pedrini, ${ }^{1}$ Alexandra Seewann ${ }^{2}$ Kirsten A Bennett, ${ }^{1}$ Alex J T Wood, \\ lan James, ${ }^{3}$ Jason Burton, ${ }^{1}$ Barry J Marshall, ${ }^{4}$ William M Carroll, ${ }_{1}^{1}$ Allan G Kermode ${ }^{1,3}$
}

\begin{abstract}
${ }^{1}$ Centre for Neuromuscular and Neurological Disorders, Western Australian

Neuroscience Research Institute, The University of Western Australia, Sir Charles Gairdner Hospital, QEII Medical Centre, Nedlands, Australia ${ }^{2}$ Department of Neurology, VU University Medical Center, Amsterdam, Netherlands ${ }^{3}$ Institute for Immunology and Infectious Diseases, Murdoch University, Murdoch, Australia

${ }^{4}$ The Marshall Centre for Infectious Diseases Research and Training, The University of Western Australia, QEII Medical Centre, Nedlands, Australia
\end{abstract}

\section{Correspondence to}

Professor Allan G Kermode, Centre for Neuromuscular and Neurological Disorders, Western Australian Neuroscience Research Institute, 4th Floor, A Block, QEIl Medical Centre, The University of Western Australia, Verdun St, Nedlands, W A 6009, Australia; kermode@me.com

MJFP and AS contributed equally to this study.

Received 22 September 2014 Revised 20 November 2014 Accepted 30 November 2014 Published Online First 19 January 2015

\section{SLinked}

- http://dx.doi.org/10.1136/ jnnp-2014-309759

\section{CrossMark}

To cite: Fabis Pedrini MJ, Seewann A, Bennett KA, et al. I Neurol Neurosurg Psychiatry 2015;86: 603-607.

\section{ABSTRACT}

Background In recent years, a relationship between Helicobacter pylori and many disease conditions has been reported, however, studies in its relationship with multiple sclerosis (MS) have had contradictory results.

Objective To determine the association between the H. pylori infection and MS

Methods 550 patients with MS were included in the study and were matched by gender and year of birth to 299 controls. Patients were assessed for clinical and demographic parameters. An enzyme immunoassay was used to detect the presence of specific $\lg G$ antibodies against $H$. pylori in the serum sample of both groups.

Results $H$. pylori seropositivity was found to be lower in the patients with MS than in controls ( $16 \%$ vs $21 \%$ ) with the decrease pertaining to females (14\% vs $22 \%$, $\mathrm{p}=0.027$ ) but not males ( $19 \%$ vs $20 \%, \mathrm{p}=1.0)$. When adjusted for age at onset, year of birth and disease duration, H. pylori seropositive females presented with a lower disability score than seronegative females $(p=0.049)$, while among males the reverse was true $(p=0.025)$. There was no significant association between H. pylori seropositivity and relapse rate.

Conclusions Our results could reflect a protective role of $H$. pylori in the disease development. However, it may be that $H$. pylori infection is a surrogate marker for the 'hygiene hypothesis', a theory which postulates that early life infections are essential to prime the immune system and thus prevent allergic and autoimmune conditions later in life. The fact that the association between $\mathrm{H}$. pylori seropositivity and MS risk was seen almost exclusively in females requires further investigation.

\section{INTRODUCTION}

Multiple sclerosis (MS) is a heterogeneous, immune-mediated demyelinating disease of the central nervous system. ${ }^{1}$ Pathogenesis involves a complex interaction between genetic and environmental factors. ${ }^{2-6}$ Recently, many studies indicated a steady increase in autoimmune disease incidence in developed countries, which could suggest that the concurrent decline in infectious disease prevalence might explain the increased autoimmune disease incidence. ${ }^{78}$ About half of the world's population is infected with Helicobacter pylori. ${ }^{9}$ $H$. pylori infection is more prevalent in developing countries, while the incidence is decreasing in Western countries. The lower rate of infection with $H$. pylori in the West is largely attributed to higher hygiene standards and widespread use of antibiotics. ${ }^{10}$
While the majority of $H$. pylori hosts remain asymptomatic, infection with it has a role in chronic gastritis, peptic ulcer disease, low-grade gastric mucosa-associated lymphoid tissue lymphoma and non-cardia gastric adenocarcinoma. ${ }^{11-13}$ H. pylori may also play a role in some extragut conditions. ${ }^{14}$ Paradoxically, there are many studies reporting benefits of infection, including a reduction in the likelihood of developing atopic disorders later in life if infected with $H$. pylori in childhood. ${ }^{15}$ Relatively unclear is any association it may have with a potentially autoimmune demyelinating condition such as MS.

The contradictory findings concerning the association between $H$. pylori infection and MS based on low numbers of patients ${ }^{16-21}$ led us to study a large group of Western Australian patients.

\section{METHODS}

\section{Research participants}

A total of 550 Caucasian patients enrolled into the Perth Demyelinating Disease Database (PDDD) ${ }^{22}$ with a diagnosis of MS according to the McDonald criteria $^{23}$ were included in the study. The study population included 412 (75\%) female cases and 138 (25\%) male cases. Serum samples were collected from these patients between the years of 2007 and 2011 with an average age at time of collection of 47.7 (range 15-83). Patients with MS were matched based on gender and year of birth (within 2 years) to the control group consisting of 299 individuals of Anglo-Celtic descent from the Busselton Community Health Study (BCHS). The study protocol was approved by the Sir Charles Gairdner Hospital Human Research Ethics Committee, and informed consent was obtained from all participants in the PDDD cohort. Approval for use of control samples was provided by the BCHS ethics committee.

\section{Data recorded}

Patients were assessed in the MS clinic by the same two neurologists (AGK and WMC) at the time of collecting samples. Data recorded included: gender, age at onset of initial symptoms, disease duration and clinical course, relapse rate, and Extended Disability Status Scale (EDSS) score at the last visit.

\section{Serology}

Anti-H. pylori serology was performed on patients and on controls at the PathWest Automated Serology Laboratory at the Queen Elizabeth II Medical Centre. An enzyme immunoassay was used 
to detect the presence of specific IgG antibodies (Ab) to H. pylori in the test sample according to the manufacturer's protocol using a BEP2000 system (Enzygnost, Germany). Briefly, serum samples were diluted in a ratio 1:20 using sample buffer then added to the test plate containing inactivated $H$. pylori antigen. Sample buffer was added along with positive and negative controls and the plates were incubated at $37^{\circ} \mathrm{C}$ for 30 min. After washing, rabbit antihuman peroxidase-IgG Ab was added and samples were incubated for $30 \mathrm{~min}$ at $37^{\circ} \mathrm{C}$. Following washing, colourimetric substrate was added and samples were incubated at $20^{\circ} \mathrm{C}$ for $30 \mathrm{~min}$ in the dark. The reaction was terminated by adding stopping solution. The test plates were read at $450 \mathrm{~nm}$ with a reference wavelength of $650 \mathrm{~nm}$. A positive result for anti-H. pylori Ab was accepted for samples with a concentration greater than $10 \mathrm{U} / \mathrm{mL}$.

\section{Statistical analysis}

Comparisons of cases and population-based controls matched $1: 1$ on the basis of gender and year of birth were carried out via an exact binomial test on the paired differences and also using conditional logistic regression to incorporate additional covariates. Within the cases, associations of seropositivity rates with multiple covariates were assessed by logistic regression. The EDSS from the last visit was analysed by censored Gaussian regression to accommodate six censored values whose lower bounds only were available. EDSS values were approximately proportional to the square roots of disease duration, hence analyses were based on the ratios EDSS/sqrt (Disease Duration). Analyses were carried out using TIBCO Spotfire S+8.2 (TIBCO Software, Inc, Palo Alto, California, USA).

Table 1 Demographics and clinical features of patients with MS and $\mathrm{HC}$ from Western Australia

\begin{tabular}{|c|c|c|}
\hline Characteristics & $\begin{array}{l}\text { Mean } \pm \text { SD or number } \\
\text { (\%) of patients with } \\
\text { MS ( } n=550)\end{array}$ & $\begin{array}{l}\text { Mean } \pm \text { SD or } \\
\text { number }(\%) \text { of HC } \\
(n=299)\end{array}$ \\
\hline \multicolumn{3}{|l|}{ Sex } \\
\hline Females & $412(75)$ & $218(73)$ \\
\hline Males & $138(25)$ & $81(27)$ \\
\hline Ratio F/M & $3: 1$ & $2.7: 1$ \\
\hline Age at disease onset, years & $35.4 \pm 10.9(10-67)$ & $N / A$ \\
\hline Females & $34.9 \pm 10.6(10-67)$ & \\
\hline Males & $37.2 \pm 11.9(12-64)$ & \\
\hline $\begin{array}{l}\text { Age at sample collection, } \\
\text { years }\end{array}$ & $47.7 \pm 12.4(15-83)$ & $43.7 \pm 8.4(23-69)$ \\
\hline Females & $47.5 \pm 12.2(19-83)$ & $43.7 \pm 8.6(23-69)$ \\
\hline Males & $48.4 \pm 12.9(15-79)$ & $43.8 \pm 7.9(29-64)$ \\
\hline Primary progressive MS & & $\mathrm{N} / \mathrm{A}$ \\
\hline Females & $19(4.6)$ & \\
\hline Males & $20(14.5)$ & \\
\hline \multicolumn{3}{|l|}{ Not Primary progressive MS } \\
\hline Females & $393(95.4)$ & \\
\hline Males & $118(85.5)$ & \\
\hline \multicolumn{3}{|l|}{ Helicobacter pylori } \\
\hline \multicolumn{3}{|l|}{ Females } \\
\hline Positive & $50(12.1)$ & $48(22.0)$ \\
\hline Negative & $362(87.9)$ & $170(78.0)$ \\
\hline \multicolumn{3}{|l|}{ Males } \\
\hline Positive & $23(16.5)$ & $16(19.8)$ \\
\hline Negative & $116(84.5)$ & 65 (81.2) \\
\hline
\end{tabular}

RESULTS

Frequency of $\boldsymbol{H}$. pylori seropositivity in patients with MS and healthy controls

Demographic and clinical data of patients with MS and healthy controls is presented in table 1 .

The rate of $H$. pylori seropositivity in the 299 matched cases was marginally lower than among the controls $(p=0.056$ unadjusted, $\mathrm{p}=0.045$ adjusted for gender). However, the differences in $H$. pylori seropositivity pertained almost exclusively to the females $(p=0.03)$, while there was no significant difference in cases versus controls for the males $(p=1.0$; figure $1 \mathrm{~A})$. Comparison of the rates of seropositivity between the matched and non-matched cases did not find any significant differences after adjusting for gender $(p=0.2)$, nor was there any difference in the male/female ratio between matched and unmatched cases $(p=0.2)$. The sampling design did not allow disentanglement of the effects of age of sampling and year of birth, and there was little overlap in birth years for the non-matched cases and the controls. To increase numbers in the comparisons we therefore also created strata based on 2-year age-at-sampling intervals using all the data and carried out case-control analyses using conditional logistic regression, including only those strata in which cases and controls were both represented. In these analyses, cases had a significantly lower seropositivity rate after adjusting for gender $(p=0.005)$, with the effect again notable among the females $(p=0.002)$ and not males $(p=0.79$; figure $1 B)$.

\section{Association of $\boldsymbol{H}$. pylori seropositivity with EDSS and relapse rate}

When adjusted for age at onset and year of birth there was a significant interaction effect of gender and $H$. pylori seropositivity on the scaled EDSS/sqrt (Disease Duration) values $(\mathrm{p}=0.003)$. There was only marginal evidence of difference between the female and male seronegative cases $(p=0.066)$. However, the female seropositive cases were lower than the corresponding female seronegative cases $(p=0.049)$ while the male seropositive cases were higher than the male seronegative cases $(p=0.025)$, figure 2. There was no significant association between $H$. pylori seropositivity and relapse rate after adjustment overall $(p=0.61)$ for females $(p=0.63)$ or for males $(p=0.99)$.

\section{The rate of $\boldsymbol{H}$. pylori seropositivity is related to the primary} progressive course of MS for females

Rates of $H$. pylori seropositivity were assessed among cases for association with age at sample collection, age at onset, year of birth, gender and primary progressive (PP) status using all the case data. After adjustment for the other variables, there was a significant interaction between gender and PP status $(p=0.037)$, with a significant increase in seroprevalence rates for PP cases compared with non-PP cases among females $(p=0.026)$, but not among males $(p=0.88$; figure 3$)$.

Overall, there was no significant association among MS cases of carriage of DRB1*1501 and seroprevalence rate $(p=0.16)$. However, within the males there was a significant association $(p=0.028)$, with DRB1*1501 males having higher seroprevalence (figure 4).

\section{DISCUSSION}

A steady decrease in $H$. pylori infection has been observed in most populations in recent decades, more accentuated in wealthy Western countries. The prevalence of $H$. pylori infection in Australian adults is lower than that in other developed countries. The overall seroprevalence of $H$. pylori infection in 

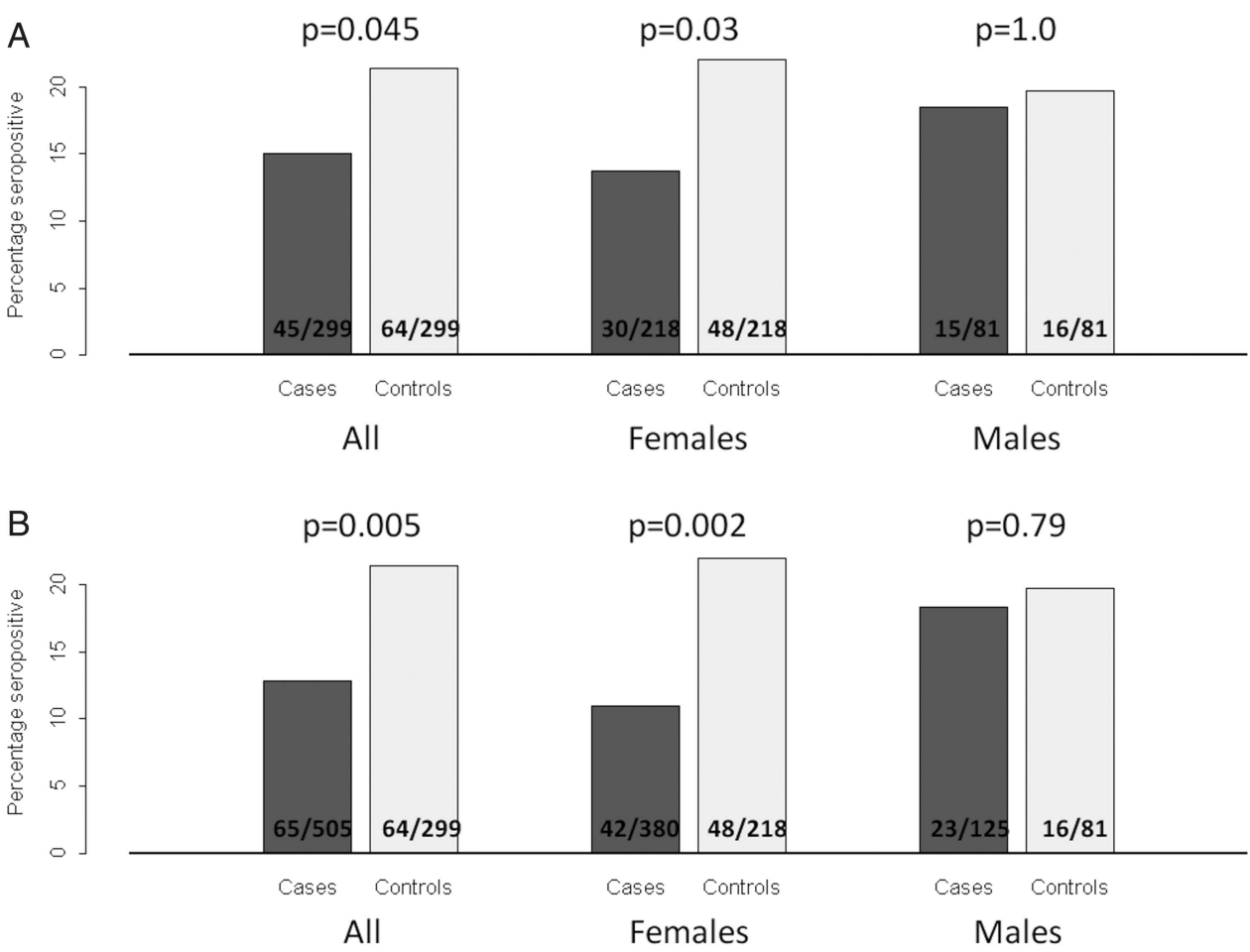

Figure 1 Frequency of Helicobacter pylori seropositivity in patients with multiple sclerosis and healthy controls. (A) $H$. pylori seropositivity rate was significantly lower in cases than in healthy controls $(p=0.045)$ when matched by year of birth. The difference was significant in females $(p=0.03)$ but not in males $(p=1.0)$. (B) H. pylori seropositivity was overall significantly lower in cases $(p=0.005)$ than in controls when matched by age at sample collection. The effect was significant among females $(p=0.002)$ but not males $(p=0.79)$. * $p$ Values based on matched groups and adjusted for gender in the overall comparison.

Australia in 2002 was $15.1 \%$, with no statistical difference between genders. ${ }^{24} \mathrm{H}$. pylori infection is most common among those living in areas of socioeconomic disadvantage or those who were born overseas. ${ }^{25}$

The present study demonstrates that H. pylori infection is lower in female patients with MS than in healthy controls. It is important to point out that the incidence of MS is increasing over the last decades, and this is mainly driven by an increase of MS in women, resulting in a higher female/male $(\mathrm{F} / \mathrm{M})$ rate. In this study, the F/M ratio was $2.7 / 1$ (matched cases) or 3/1 (nonmatched cases).
In our study, we enrolled 550 patients with conventional MS and 299 carefully selected community-based controls. Such numbers represent the largest number ever evaluated in regard to association between $H$. pylori infection and MS. Rates of H. pylori seropositivity were assessed among cases for association with disease course. We found evidence of an association with PP course, but interestingly, again only among females. Previous much smaller studies on the association between H. pylori and MS were contradictory. Some authors stated strong positive association between $H$. pylori infection and MS, ${ }^{18}$ and others found that patients with MS presented with
Figure 2 Association of Helicobacter pylori seropositivity and EDSS score/sqrt (Disease Duration). On adjustment for age and year of birth, EDSS score/sqrt (Disease Duration) significantly interacted with gender and $H$. pylori seropositivity $(p=0.003)$. The effect was present in females in whom seropositivity was significantly higher than seronegativity $(p=0.049)$, and males in whom seropositivity was significantly lower than seronegativity $(p=0.025$; EDSS, Extended Disability Status Scale; F-sero-neg, female seronegative; F-sero-pos, female seropositive; M-sero-neg, male seronegative; M-sero-pos, male seropositive; sqrt, square root).

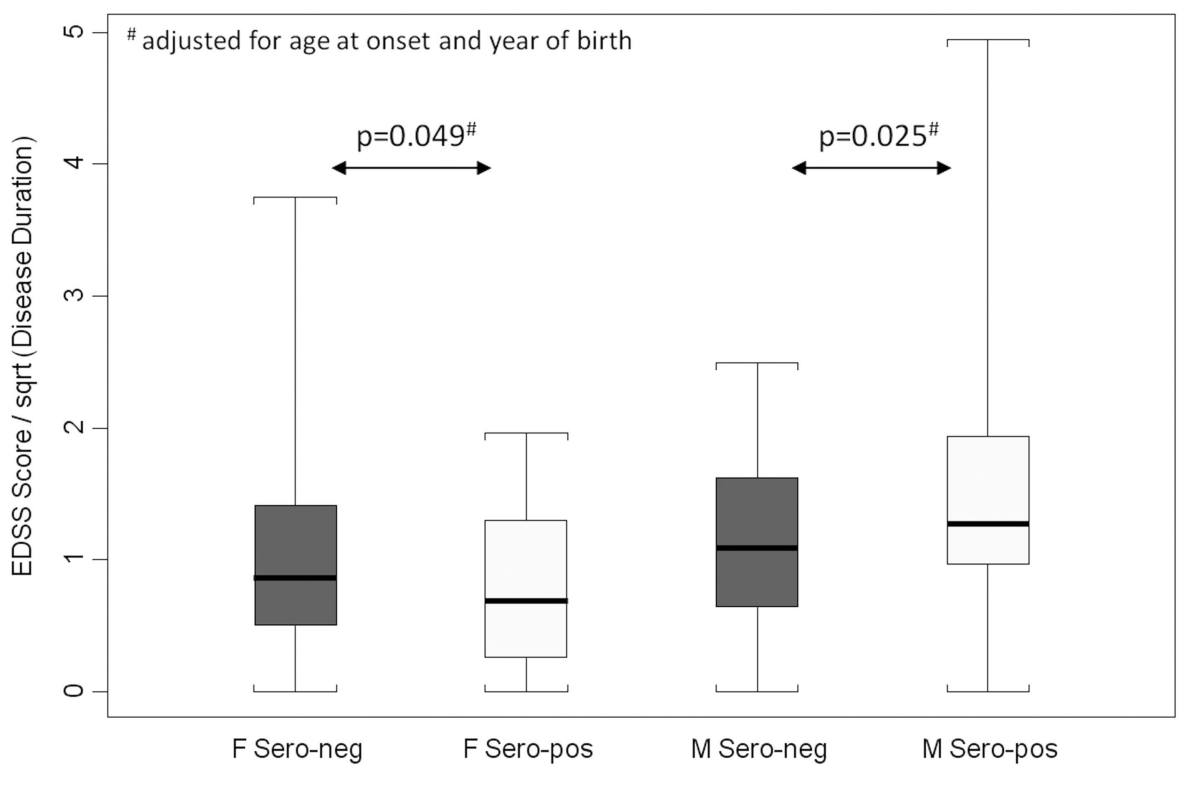


Figure 3 Frequency of Helicobacter pylori seropositivity in primary progressive (PP) and not PP multiple sclerosis cases. Rates of $H$. pylori seropositivity were assessed among cases for association with age at sample collection, age at onset, year of birth, gender and PP status using all the case data. After adjustment for the other variables $H$. pylori seropositivity was associated with PP disease course overall $(p=0.082)$. When broken down by gender the effect was significant among females $(p=0.026)$, but not among males $(p=0.88)$.

lower H. pylori seroprevalence than healthy controls, ${ }^{16} 1720$ while some did not find any correlation. ${ }^{19} 2126$ Our large controlled study indicates a difference in $H$. pylori seropositivity between Western Australian patients with MS and healthy controls, but only among females. Our observations suggest that infection with $H$. pylori can be a protective factor against MS risk in Western Australian females.

A protective role of $H$. pylori infection is consistent with the hygiene hypothesis, which postulates that infections in early life may prime the immune system, and suppress allergic and autoimmune conditions in later life. ${ }^{27}$ Also in favour of a protective effect is the finding that infection with $H$. pylori can reduce the prevalence of allergic disorders by $30 \%$ compared with noninfected individuals, ${ }^{15}$ and likewise infection with a parasite could be a protective factor in MS. ${ }^{6}$ One simplistic explanation for this may be that a bacterial infection can promote a $\mathrm{T}$ helper type 1 (Th1) response, shifting the balance away from Th2 cells. Given that it is Th2 cells that are involved in hypersensitivity and atopic disorders, it is reasonable to conclude that this shift may be responsible for the aforementioned reduction in incidence of atopic disorders. Additionally, a study by Lynch et al ${ }^{28}$ found that individuals infected with parasites experienced recurrence of allergic symptoms when treated with antiparasitic agents. These findings lend weight to the hypothesis that past infections have a protective effect on autoimmune disease. It has already been hypothesised that should $H$. pylori be confirmed to have a protective effect against MS, $H$. pylori pharmaceutical products could provide a novel means of treatment via nanoparticle delivery to neuron-specific targets. ${ }^{29}$

In a number of studies it has been shown that the major histocompatibility complex class II genes are important genetic factors that influence MS susceptibility. In Caucasians, the HLA-DRB1*1501 allele is strongly associated with $\mathrm{MS}^{30},{ }^{30}$ but we found little evidence of association between HLA-DRB1*1501 allele and H. pylori seroprevalence that was limited to males only.

Figure 4 Association between Helicobacter pylori seropositivity and a carriage of DRB1*1501. Significant association between DRB1*1501 carriage rate and $H$. pylori seroprevalence was seen in males only $(p=0.028)$, but not the group as a whole $(p=0.16)$. * $p$ Values exact.
In summary, H. pylori infection is associated with a slight but significant reduction of MS risk in Western Australian women. Prior publication differences concerning the association between H. pylori infection and disease risk could be due to differences in the size of sample groups, differences in ethnicity, time of infection, or perhaps different subtypes of MS. A lower frequency of $H$. pylori infection might be a reflection of good sanitation in developed countries. One weakness of our study might be that we were not able to control for country of birth and ethnicity. This could result in spurious findings. However, most of our patients with MS were born in Australia, UK or other Western countries. Even if we did not have this information for our community-based controls, it is known that Western Australia has a relatively stable population, largely of Anglo-Celtic descent, thus it would be unlikely that we would find significant differences in country of birth between patients with MS and controls. Future studies could address this issue. The differences observed here were not due to changes in behaviour after MS onset, as infection usually occurs in early childhood. The reason why the influence of $H$. pylori infection on MS risk affects females only is unknown and warrants further investigation.

Acknowledgements The authors thank all participants in the Perth Demyelinating Disease cohort as well as the Busselton cohort participants for their involvement.

Contributors MJFP contributed in the drafting and revising of the manuscript, and in the analysis and interpretation of the data. AS and KAB contributed in the analysis and interpretation of data, and in the revising of the manuscript. AJTW was involved in the clinical data collection and revising of the manuscript. IJ participated in the statistical analysis and manuscript preparation, and in the revising of the manuscript. JB was involved in the consultation for neurology patient care, and in the revising of the manuscript. BJM performed critical revision of the manuscript. WMC was involved in the consultation for neurology patient care, and in the critical revision of the manuscript. AGK was involved in the consultation for neurology patient care, and contributed in the study conception and designing, analysis and interpretation of data, and critical revision of the manuscript.

Competing interests BJM has received funding from The University of Western Australia and from NHMRC Sir Macfarlane Burnet Fellowship grant (572723). He is

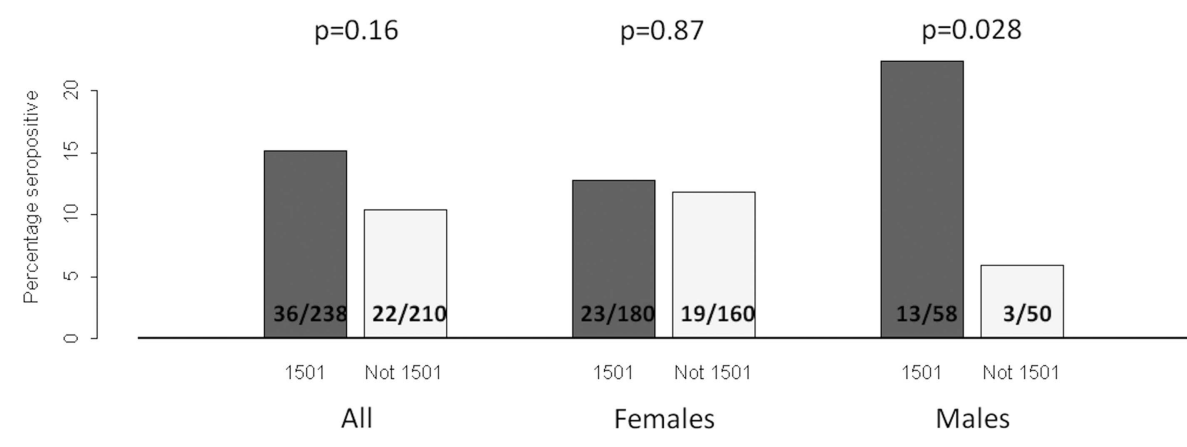


a substantial owner of Ondek, a company attempting to develop biological delivery systems from Helicobacter. WMC received speaker honoraria and membership of scientific advisory boards from Bayer Schering, Novartis, Merck Serono, Sanofi, and Biogen-Idec. AGK received speaker honoraria and scientific advisory board fees from Bayer, Biogen-Idec, Novartis, Sanofi-Aventis, Merck, Sanofi-Genzyme and Innate Immunotherapeutics. Dr KAB and Dr AJTW were supported by Multiple Sclerosis Research Australia Vacation Scholarships.

Patient consent Obtained.

Ethics approval Sir Charles Gairdner Hospital Human Research Ethics Committee.

Provenance and peer review Not commissioned; externally peer reviewed.

\section{REFERENCES}

1 Compston A, McDonald I, Noseworthy J, et al. McAlpine's multiple sclerosis. 4th edn. Elsevier, 2006.

2 Lipton $\mathrm{HL}$, Liang $\mathrm{Z}$, Hertzler $\mathrm{S}$, et al. A specific viral cause of multiple sclerosis: one virus, one disease. Ann Neurol 2007;61:514-23.

3 Compston A, Coles A. Multiple sclerosis. Lancet 2008;372:1502-17.

4 Ebers GC. Environmental factors and multiple sclerosis. Lancet Neurol 2008;7:268-77.

5 Ramagopalan SV, Dobson R, Meier UC, et al. Multiple sclerosis: risk factors, prodromes, and potential causal pathways. Lancet Neurol 2010;9:727-39.

6 Correale J, Farez M. Association between parasite infection and immune responses in multiple sclerosis. Ann Neurol 2007;61:97-108.

7 Poser S, Stickel B, Krtsch U, et al. Increasing incidence of multiple sclerosis in South Lower Saxony, Germany. Neuroepidemiology 1989;8:207-13.

8 Farrokhyar F, Swarbrick ET, Irvine EJ. A critical review of epidemiological studies in inflammatory bowel disease. Scand J Gastroenterol 2001;36:2-15.

9 Marshall BJ, Warren JR. Unidentified curved bacilli in the stomach of patients with gastritis and peptic ulceration. Lancet 1984;1:1311-15.

10 Malaty HM. Epidemiology of Helicobacter pylori infection. Best Pract Res Clin Gastroenterol 2007;21:205-14.

11 Cover TL, Blaser MJ. Helicobacter pylori in health and disease. Gastroenterology 2009:136:1863-73.

12 Suerbaum S, Michetti P. Helicobacter pylori infection. N Engl J Med 2002:347:1175-86.

13 Suzuki H, Marshall BJ, Hibi T. Overview: Helicobacter pylori and extragastric disease. Int J Hematol 2006:84:291-300.

14 Cremonini F, Gasbarrini A, Armuzzi A, et al. Helicobacter pylori-related diseases. Eur J Clin Invest 2001:31:431-7.
15 McCune A, Lane A, Murray L, et al. Reduced risk of atopic disorders in adults with Helicobacter pylori infection. Eur I Gastroenterol Hepatol 2003;15:637-40.

16 Wender M. [Prevalence of Helicobacter pylori infection among patients with multiple sclerosis]. Neurol Neurochir Pol 2003;37:45-8.

17 Li W, Minohara M, Su JJ, et al. Helicobacter pylori infection is a potential protective factor against conventional multiple sclerosis in the Japanese population. J Neuroimmunol 2007;184:227-31.

18 Gavalas E, Kountouras J, Deretzi G, et al. Helicobacter pylori and multiple sclerosis. J Neuroimmunol 2007;188:187-9; author reply 90.

19 Li W, Minohara M, Piao H, et al. Association of anti-Helicobacter pylori neutrophil-activating protein antibody response with anti-aquaporin-4 autoimmunity in Japanese patients with multiple sclerosis and neuromyelitis optica. Mult Scler 2009:15:1411-21.

20 Mohebi N, Mamarabadi M, Moghaddasi M. Relation of helicobacter pylori infection and multiple sclerosis in Iranian patients. Neurol Int 2013:5:31-3.

21 Yoshimura S, Isobe N, Yonekawa T, et al. Genetic and infectious profiles of Japanese multiple sclerosis patients. PLoS One 2012;7:e48592.

22 Wu JS, Zhang MN, Carroll WM, et al. Characterisation of the spectrum of demyelinating disease in Western Australia. I Neurol Neurosurg Psychiatry 2008;79:1022-6.

23 McDonald WI, Compston A, Edan G, et al. Recommended diagnostic criteria for multiple sclerosis: guidelines from the International Panel on the diagnosis of multiple sclerosis. Ann Neurol 2001;50:121-7.

24 Moujaber T, Maclntyre CR, Backhouse J, et al. The seroepidemiology of Helicobacter pylori infection in Australia. Int I Infect Dis 2008;12:500-4.

25 Pandeya N, Whiteman DC. Australian Cancer Study. Prevalence and determinants of Helicobacter pylori sero-positivity in the Australian adult community. J Gastroenterol Hepatol 2011;26:1283-9.

26 Long Y, Gao C, Qiu W, et al. Helicobacter pylori infection in neuromyelitis optica and multiple sclerosis. Neuroimmunomodulation 2013;20:107-12.

27 Cremonini F, Gasbarrini A. Atopy, Helicobacter pylori and the hygiene hypothesis. Eur I Gastroenterol Hepatol 2003;15:635-6.

28 Lynch NR, Hagel I, Perez M, et al. Effect of anthelmintic treatment on the allergic reactivity of children in a tropical slum. J Allergy Clin Immunol 1993:92:404-11.

29 Pezeshki MZ, Zarrintan S, Zarrintan MH. Helicobacter pylori nanoparticles as a potential treatment of conventional multiple sclerosis. Med Hypotheses 2008;70:1223.

30 Wu JS, James I, Qiu W, et al. HLA-DRB1 allele heterogeneity influences multiple sclerosis severity as well as risk in Western Australia. J Neuroimmunol 2010:219:109-13. 